\title{
Corela
}

Cognition, représentation, langage

13-2 | 2015

Vol. $13, n^{\circ} 2$

\section{Vers un Pidgin-English jeune en zone anglophone du Cameroun?}

\section{Céphanie Mirabelle Gisèle PIEBOP}

\section{(2) OpenEdition}

\section{Journals}

Édition électronique

URL : http://journals.openedition.org/corela/4132

DOI : $10.4000 /$ corela.4132

ISSN : 1638-573X

\section{Éditeur}

Cercle linguistique du Centre et de I'Ouest - CerLICO

Référence électronique

Céphanie Mirabelle Gisèle PIEBOP, « Vers un Pidgin-English jeune en zone anglophone du Cameroun? », Corela [En ligne], 13-2 | 2015, mis en ligne le 01 décembre 2015, consulté le 01 mai 2019. URL : http://journals.openedition.org/corela/4132 ; DOI : 10.4000/corela.4132

Ce document a été généré automatiquement le 1 mai 2019.

\section{(c) (i) (2)(2)}

Corela - cognition, représentation, langage est mis à disposition selon les termes de la licence Creative Commons Attribution - Pas d'Utilisation Commerciale - Partage dans les Mêmes Conditions 4.0 International. 


\title{
Vers un Pidgin-English jeune en zone anglophone du Cameroun?
}

\author{
Céphanie Mirabelle Gisèle PIEBOP
}

\section{Introduction}

1 Dans un environnement essentiellement plurilingue comme celui du Cameroun, les contacts qu'entretiennent les différentes langues entre elles les amènent à s'altérer. Dans cette jungle linguistique, on assiste à des cas de figure où des langues assument des statuts, forces et pouvoirs égaux ou différents. En fonction des types de relations qu'elles entretiennent, certaines peuvent se neutraliser ou au contraire devenir dominantes. Les langues en voie d'être dominées peuvent en quelque sorte développer des stratégies visant à se revitaliser, et à contrer l'hégémonie des langues dominantes, ou alors accepter cette domination et d'une certaine manière « coopérer » pacifiquement. Mais quelle que soit la situation, les langues en contact ne sortent jamais indemnes de ces conflits. Qu'elles soient dominantes ou non, leur structure est toujours impactée, même lorsqu'il s'agit d'un parler composite du calibre du Pidgin-English, aussi appelé kamtok, la langue véhiculaire la plus populaire du Cameroun. Même à ce titre, le kamtok n'échappe pas au dynamisme ambiant, car l'insécurité linguistique permanente dans laquelle se trouvent ses locuteurs les amène à le modifier afin de mieux l'adapter aux besoins d'expression. Ainsi, se développe-t-il depuis quelques temps dans les zones anglophones du Cameroun en général, une variante du Pidgin-English très utilisée par les jeunes locuteurs.

2 Quelle est la singularité de cette variante du pidgin initial ? C'est la question à laquelle veut répondre la présente analyse. Elle vise à mettre en relief les changements qui distancient désormais le kamtok du nouveau pidgin. Elle montre comment les phénomènes observés illustrent le variationnisme que Labov (1976 : 26) considère comme « un examen de variation sociale et stylistique sous l'angle du degré de conscience que les locuteurs ont des variantes présentes dans leur parler et dans celui des membres de leur communauté. » 
3 Tout au long de l'analyse, l'approche sociolinguistique dans son ensemble permettra de décrire les traits qui démarquent le Pidgin-English «jeune » du pidgin courant et de les comparer, dans le but de mieux cerner leurs contours. Les données de ce travail examinant la question du dynamisme en contexte plurilingue camerounais, ont été recueillies à partir des productions écrites des élèves de form 5 , Lower et Upper Sixth du Lycée Bilingue de Molyko-Buea, et bien à travers des productions orales et écrites recueillies sur les campus de l'Université de Buéa, de l'Ecole Nationale Supérieure des Travaux Publics annexe de Buea, dans les rues, les taxis, le voisinage, etc, à l'insu de leurs énonciateurs (86 au total), afin d'éviter que les résultats ne soient biaisés.

\section{Brève revue du Pidgin-English}

4 Le Pidgin-English, ou kamtok, ou encore CamP (Cameroon Pidgin) est un idiome qui est né au dix-huitième siècle quand l'anglais britannique est entré en contact avec les langues bantoues de la côte ouest africaine. Cette nouvelle langue prit très vite de l'expansion et joua d'ailleurs un rôle incontestable dans les interactions communicationnelles informelles et parfois formelles, entre les locaux d'une part, et entre les locaux et les puissances coloniales, à un niveau national et même international d'autre part. On peut même dire qu'en cette période, elle faisait office de langue officielle, nonobstant son statut illégal et non normé. Tabi Manga (2000: 17) rappelle d'ailleurs que c'est cette langue qui permit la communication qui aboutit à la signature du traité d'annexion du Cameroun entre l'Allemagne et les chefs Douala. En tant que langue la plus parlée au Cameroun, le Pidgin-English fait de nos jours l'objet de nombreux débats, surtout lorsqu'il s'agit de déterminer une langue nationale pour l'ensemble des Camerounais. En fait, certains, le gouvernement aux premières loges, voient en cette langue un danger pour la bonne connaissance de l'anglais, d'autres, et c'est le cas de Ngijol (1964), Todd (1983:169) et $\mathrm{Echu}^{1}$, pensent que le Pidgin-English est particulièrement bienvenu pour assumer la fonction d'idiome national.

\section{Le Pidgin-English des zones anglophones}

5 Le kamtok des régions francophones n'est pas identique à celui des zones anglophones, objet de présentes analyses. On peut alors se demander à quels niveaux reposent les différences entre ces parlers frères. En effet, il existe des différences phonétiques et lexicales, ce qui complique l'intercompréhension. Selon une certaine doxa, le PidginEnglish parlé par les anglophones peut apparaître comme une sorte de dialecte par rapport à l'anglais, un anglais de brousse, sa variété basse ou low, si l'on veut parler en terme de diglossie, car il demeure dans une continuité interlinguistique. Par contre, le Pidgin-English parlé par les francophones est dans un rapport de discontinuité interlinguistique avec le français. Il existe également des différences notoires dans la façon de parler qui peuvent permettre de voir en un énoncé les traces du Pidgin-English anglophone ou francophone. Par exemple, l'énoncé «ami donne-moi une pièce de cent francs " peut se traduire par «membre gi mi pièce fo de » (pidgin francophone), ou bien «brother gi mi one coin " (pidgin anglophone). De même, « mburu sep fo pe location no de » correspondrait à « money sef fo pe rent no de » (« il n'y a pas d'argent pour payer le loyer »). 
6 Ainsi, compte tenu des variations intralinguistiques, et des considérations de locuteurs qui différencient ces deux formes par un élément de caractérisation, on peut affirmer que ces deux formes de pidgin sont une réalité au Cameroun. C. de Féral (1989: 42-43) résume plus clairement cette variation: "les réalisations phonétiques des locuteurs de la zone anglophone sont plus proches de l'anglais standard que celles des Francophones ", et elle ajoute encore plus loin que «les degrés de connaissance de l'anglais» feraient que «les Anglophones non scolarisés parlent [...] une variété plus proche du P.E.F. que les Anglophones scolarisés ». Dans la même lancée, B.S. Chumbow et A.S. Bobda (2000) présentent un Francophone Pidgin English vu comme «the variety spoken by francophone Cameroonians (FPE) [which] comprises several French words » $(2000: 55)$.

7 En rapport avec le cas du kamtok parlé en zone anglophone, il a été notifié tel qu'exposé plus haut qu'en dehors de son usage habituel, il existe une autre variante très affectionnée par les jeunes, et qui se démarque de plus en plus des usages initiaux du Pidgin-English. Mais en quoi consistent ces démarcations? La section suivante prend en charge cette problématique.

\section{Thématique et traits spécifiques au Pidgin-English jeune}

Lorsque la majeure partie des jeunes des zones anglophones et de Buea en particulier parlent le Pidgin-English, ils abordent le plus souvent des thèmes qui leur sont spécifiques et qui reflètent mieux leur vision du monde. A ce sujet, un questionnaire a été adressé aux $2 / 3$ du public cible, c'est-à-dire 31 locuteurs. Il se dégage des récurrences de leurs réponses que les jeunes abordent dans leurs interactions des sujets en relation avec les préoccupations d'ordre académique, les relations amoureuses et amicales, les passetemps (le football pour les garçons, la beauté pour les filles, l'habillement en vogue, la gastronomie, les idoles), l'argent, les fêtes et autres réjouissances nocturnes, les ambitions, les projets futurs, l'après-école, les problèmes du quotidien (santé, problèmes familiaux...), les moyens de locomotion, les petites affaires liées aux gadgets les plus en vue (ordinateurs, cartes mémoires, i-pods, puces, tablettes...)

9 Par ailleurs, les thématiques abordées par les jeunes ont également des spécificités, dans la mesure où ces derniers créent des changements dans leur langage qu'ils n'auraient pas pris la peine d'opérer en face de leurs parents ou des marchants pidginophones d'un certain âge. Ces changements recouvrent pour la plupart les domaines de l'emprunt, de la proso-/ phonologie, des connotations des expressions idiomatiques, de la dérivation, de la transcatégorisation, des périphrases, des calques, des onomatopées, etc.

\subsection{Les emprunts}

10 D'après Piebop (2014: 146), l'emprunt linguistique est un phénomène dû au contact de langues «qui, par un processus néologique, se définit par l'intégration dans une langue d'un procédé ou d'un élément d'une langue étrangère pour combler une lacune métalinguistique, par goût de l'exotisme ou par snobisme. » Autrement dit, il s'agit d'un processus d'intégration qui doit s'analyser, soit à travers le sujet en contact avec les usagers d'une autre langue, sujet bilingue donc qui introduira dans ses usages un élément (phonologique, syntaxique, sémantique, lexical...) de la langue avec laquelle il est en 
contact, soit par mode progressif d'introduction de l'élément emprunté dans le système de la langue. L'emprunt est donc au carrefour des langues et des cultures. Mieux, c'est par ce phénomène de contact et de compénétration que les langues évoluent, se transforment et s'enrichissent substantiellement. On observera comme termes étrangers au système du Pidgin-English, ceux venant de l'anglais savant, du français, du camfranglais, des langues maternelles locales, des langues nigérianes et ivoiriennes.

\subsubsection{L'emprunt à l'anglais}

11 Le Pidgin-English est en général un parler qui se distingue par la grande flexibilité de son système linguistique, qui donne pratiquement à chacun de ses locuteurs la latitude d'agencer ses structures phrastiques selon son inspiration. Pour cette raison, il n'a aucun égard pour les règles de grammaire de la langue anglaise dont il peut être considéré comme une variété « basse ». Pourtant, le Pidgin-English jeune a ceci de particulier qu'au lieu de s'éloigner de l'anglais, il s'en rapproche plutôt. Les emprunts sont centrés au niveau du vocabulaire spécialisé ou connoté, comme le présentent ces illustrations :

1. Ma friend, a tell you sé a don bi admitted for my master programm (Mon ami j'ai été admise à suivre un programme de master)

2. A si you yesterday wit that ya milky baby (Je t'ai vu hier avec une séduisante petite amie)

3. Our maths lecturer di sell handout for up to 5 000. A say man go die inside this UB

(Notre enseignant de mathématique vend ses fascicules au prix élevé de 5000 Frs. Quelqu'un va mourir dans cette université de Buéa $=$ Ce n'est pas un endroit pour les pauvres.).

Les termes admitted et master programm tels qu'ils apparaissent ici sont des emprunts à l'anglais savant. Ils sont spécialisés dans le domaine de la scolarité universitaire surtout. Pour ce qui est de milky, c'est un adjectif qui au lieu de référer à quelque chose qui contient beaucoup de lait ou encore à la couleur blanche lactée, est ici en emploi connotatif, puis qu'il signifie séduisante. En outre, admitted apparait sous la forme d'un participe passé. Ces termes relèvent dès lors du grammar, c'est-à-dire de la grammaire pure, qui du reste est anxiogène pour un locuteur pidginophone et qui est quasi absente en pigdin pur. En pidgin simple, admitted aurait été remplacé par pass exam/class, de même que milky baby aurait été remplacé par fine girl friend. L'usage de ce vocabulaire savant peut se justifier par le niveau intellectuel de cette tranche de locuteurs qui pour la plupart sont des étudiants d'universités et de grandes écoles.Voilà pourquoi l'on ne serait plus surpris de retrouver dans le corpus des occurrences comme lecturer et handout du troisième exemple, qui appartiennent également au champ lexical de l'éducation dans l'enseignement supérieur en général, étant donné que lecturer désigne l'enseignant et handout le manuel/ fascicule.

Il avait déjà été signalé par de nombreux linguistes que le Pidgin-English évolue, et tend à s'angliciser davantage au fils du temps. Les études de Ngefac et Sala (2006: 226) relèvent ceci: « particularities that characterized CamP (Cameroon Pidgin) in the 1960s are hardly any longer perceivable in the speech of today's speakers of CamP who have been increasingly exposed to English. » Cela amène les auteurs à placer ces deux langues à une confluence qui à son tour déterminera un continuum. Mais la particularité ici repose sur le fait que les emprunts utilisés par les jeunes pidginophones relèvent le plus souvent du registre savant. Car il est en général composé de tournures recherchées et d'unités lexicales spécialisées dans les domaines qu'ils côtoient au quotidien dans les 
amphithéâtres et qui par conséquent ne peuvent qu'influencer leurs interactions en pidgin. Cette profusion des termes anglais dans le discours constitue également un trait de démarcation entre ce pidgin des jeunes anglophones et celui des francophones (on l'a déjà signalé comme moins dominé par l'anglais). Il s'agit d'un phénomène compréhensible dans un contexte plurilingue comme celui du Cameroun, où la «guerre des langues » est sans cesse présente, car l'anglais constitue la première langue officielle des locuteurs, c'est-à-dire le superstrat qui bénéficie de tous les privilèges. Son hégémonie se ressent même dans les discours en d'autres langues, et qui de surcroît se veulent informels. Mais en dehors de l'anglais pur, le français est également sollicité par le Pidgin-English jeune. réponses sont classées dans le jargon jeune, d'autant qu'il s'agit de deux écoliers qui conversent. En plus, s'il s'était agi du Pidgin-English pur, on aurait respectivement pu dire :

A no wel, A di sik but a di tie heart/try for go school,/ Achia! A just be want tell you sé a bi happy (di glad)/ A beg, no go wé you no ba gi me something

作 exerce également une forte influence sur leurs interactions communicationnelles du fait de sa présence aux côtés de l'anglais dans la scolarisation, la radio, la télévision, les institutions étatiques et les autres lieux d'enracinement idéologique. Très souvent, l'emploi des termes français dans les échanges pidginophones jeunes surtout leur donne le sentiment d'être des Camerounais à part entière, c'est-à-dire sachant manier à la fois le français et l'anglais (même s'ils les pidginisent consciemment ou pas) pour s'y sentir à l'aise. A ce moment, le français devient une langue prestigieuse, voire supérieure pour les jeunes pidginophones qui s'en servent.

19 En outre, une grande partie des locuteurs affectionnant l'emprunt au français se situe dans la tranche de ceux qui pour des raisons diverses entrent régulièrement en contact avec les zones ou les locuteurs francophones. Et pour montrer cette supériorité et l'efficacité de leur éloquence sur leurs co-locuteurs restés en zone anglophone, ils pratiquent le code switching et le code mixing, ainsi qu'on peut le constater dans ces exemples :

5. Tu pars où ? A beg gi ma doh first. (Où vas-tu? s'il te plait donne moi d'abord mon argent)

Ce prototype présente un cas d'alternance de code que Gumpez (1982 : 485) définit comme «la juxtaposition à l'intérieur d'un même échange verbal, de passages où le discours 
appartient à deux sous systèmes grammaticaux. » Il convient de relever que, pour qu'il y ait alternance de code, il faudrait retrouver dans un même texte ou discours non seulement des éléments appartenants à des systèmes linguistiques différents, mais également appartenants à des phrases différentes.

Dans l'exemple, la première phrase Tu pars où ?dont la fin est signalée par le point d'interrogation est composée de termes et d'une structure appartenant au système linguistique français. Pourtant celle qui suit est identifiée comme appartenant au PidginEnglish jeune. Cette alternance entre le français et le pidgin surtout s'explique aussi par le désir des jeunes de mieux assimiler les leçons de français, ou alors de montrer qu'ils sont des bilingues accomplis, comme le veut la politique linguistique du pays.

A côté de l'alternance codique, les propos des jeune pidginophones sont aussi dominés par le code mixing qui contribue à valoriser leur identité de Camerounais imprégnés de leurs diverses langues et cultures, et également ouverts à une civilisation de l'universel. Cette correction d'une étudiante à l'endroit de son ami le montre :

6. Lecturer no say imparfait. Na passé composé they di use for recent actions. And when they di narrate recent actions like to talk about thing wé you douam yesterday, they also use connecteurs logiques like d'abord, après, ensuite, finalement. I for be na nkap so you no for confuse. (Le prof n'a pas dit l'imparfait. C'est le passé composé qui s'utilise pour décrire des actions récentes. Et lorsque l'on raconte des événements récents, ce que tu as fait hier par exemple, il faut aussi utiliser des connecteurs logiques comme d'abord, après, ensuite, finalement. S'il s'était agit d'argent, tu ne te serais pas trompé !!!!)

Cette illustration présente des mélanges de codes en ceci que ce n'est plus d'une phrase à l'autre comme avec l'alternance de code, que le code linguistique change, mais à l'intérieur même de celles-ci. La première phrase commence en pidgin (Lecturer no say) et se termine en français (imparfait). La deuxième quant à elle commence en pidgin (Na) puis mute en français (passé composé) puis bascule de nouveau en Pidgin-English (they di use for recent actions). La troisième va du pidgin (And when they di narrate recent actions like to talk about thing wé you douam yesterday, they also use) au français (connecteurs logiques), puis à l'anglais (like) et au français (d'abord, après, ensuite, finalement). La dernière entame avec le pidgin (I for be), puis suit le ghomala'a langue nationale de l'ouest (nkap) puis le pidgin (so you no for confuse). Ainsi peut-on retenir que l'alternance de codes est inter-phrastique alors que le mélange de codes est plutôt intra-phrastique. Ces phénomènes, du reste très proches, témoignent des relations qu'entretiennent les langues en contact en zone anglophone du Cameroun et surtout de la fonction identitaire qu'assume le Pidgin-English tout court. Autrement dit, les locuteurs sont influencés par un environnement plurilingue qui est également marqué par le camfranglais.

\subsubsection{L'emprunt au camfranglais}

Parler hybride, au même titre que le kamtok, le camfranglais est spécifique à la jeunesse des zones francophones et il fascine également de nombreux jeunes locuteurs anglophones, qui ne manquent pas de le faire savoir en l'utilisant dans les communications informelles.

Il n'est pas superflu de noter qu'il existe tout de même une différence entre le PidginEnglish et le camfranglais. En effet, tandis que le pidgin apparaît comme on l'a déjà signalé au $18^{\text {ème }}$ siècle, le camfranglais quant à lui ne voit le jour que dans les années 1970, ainsi 
que le signale C. de Féral (1989) au Cameroun. Il naît d'une variété de français dite "français makro", c'est-à-dire le français des voyous désireux de crypter leurs communications. Peu à peu ce parler aurait évolué, à partir d'origines peu prestigieuses et de son cercle restreint d'usagers, vers une étape intermédiaire, avant de devenir ce qu'on appelle le camfranglais, symbole identitaire d'un groupe plus large constitué d'adolescents et de jeunes adultes en cours de scolarisation ou ayant été scolarisés. Ainsi, le mot-valise camfranglais met en exergue une identité camerounaise sans distinction d'ordre social, que ne peut assumer la multitude de langues ethniques, non plus que le bilinguisme officiel français/anglais. C. de Féral (1989 : 258) voit d'ailleurs en ce parler "un objet linguistique apparemment bien délimité et plutôt socialement et linguistiquement valorisé puisque y sont réunis dans un mot valise (camfranglais ou francanglais) les noms des deux langues officielles du Cameroun. En outre, avec cam (Cameroun) dans camfranglais, s'affiche également une identité territoriale, nationale ».

Il faut signaler que le Pidgin-English des francophones diffère aussi du camfranglais dans la mesure où, parlé par toutes les tranches des populations, il a existé avant l'apparition du camfranglais et ne nécessite pas forcément de compétences linguistiques dans les langues officielles, comme c'est le cas avec le camfranglais, pour être parlé. En fait, il est même possible que ce soit le Pidgin-English des francophones qui, au cours de son évolution, a donné naissance au camfranglais.

$\mathrm{Au}$ cours du dépouillement des données, on a identifié des termes puisés dans le camfranglais. Un jeune raconte sa mésaventure à ses voisins en ces termes :

7. One day wé ngué(mé) bi di dou mi, no bi a enter ma pah hi room, a ngniè ${ }_{1}$ kolo, no bi a ting sé na ma ndolè, A ték the doh. A wan siam hi take mi for ngata. A say ngniè tochoh me so té wan bolè me. (Un jour, alors que j'étais en manque d'argent, je suis entré dans la chambre de mon père et j'ai vu 1000frs que j'ai pris. Avant de m'en rendre compte, mon père m'a amené à la police et j'y ai reçu la bastonnade de ma vie.)

En Pidgin-English normal, on pourrait remplacer nguémé par « a no bi get money », ngniè par «see» kolo par «one tohsing», doh par «money", ngata par "gateroom/police», ngniè ${ }_{2}$ par « policeman », bolè par « finish ».

Relevons que ndolè subit un glissement sémantique en camfranglais, puisqu'il n'a plus rien à voir avec le plat des Sawa. Selon son acception la plus en vogue, il renvoie à un profit, une propriété, un bien.

Comme on peut le voir, ces termes appartiennent au camfranglais, mais sont transportés dans le Pidgin-English des jeunes. A travers ces transports on comprend que le camfranglais est une langue composite identitaire très importante et en pleine expansion dans le paysage linguistique camerounais, car son domaine d'extension va au-delà de la zone francophone d'origine pour déborder en zone anglophone, où son usage a la faveur des jeunes. En dehors du camfranglais, les emprunts aux langues locales se retrouvent aussi dans le Pidgin-English jeune.

\subsubsection{L'emprunt aux langues locales}

31 Selon un constat général, ne sont convoqués dans le Pidgin-English jeune que les termes locaux les plus à la mode et les plus en rapport avec les préoccupations de ces jeunes. Ainsi en est-il de terme ndolè qui apparaît dans le contexte ci-après. Un étudiant donne l'information à son camarade sur le menu du jour au restaurant : 
8. A- They cook na ndolè today for restau we go dé ? (Le menu du jour au restau c'est

le ndolè, on y va?)

$B$ - No a no go tchop djoka dé for soirée. (Non, je me préserve pour la fête du soir) un plat de légumes douala. Il est rendu en Pidgin-English par bitter leaf. Quant à djoka il s'agit d'un mot bassa qui signifie tout simplement la fête. Il est également à signaler qu'avant d'être utilisés dans le Pidgin-English jeune, ces termes ont dû transiter par le camfranglais qui les a finalement cédés à cet autre parler qui tout comme lui, recrute en premier des locuteurs jeunes, qui sont par essence des amoureux des fêtes et autres cérémonies d'évasion. Par conséquent, ces mots n'ont d'autre choix que de migrer des langues locales vers le kamtok, en faisant une halte dans le camfranglais. Ce ne sont que quelques illustrations à titre indicatif des termes locaux empruntés par le kamtok jeune. Et à travers elles, on voit le désir de ces jeunes locuteurs de s'enraciner dans les langues et cultures endogènes et de les valoriser malgré tous les attraits et privilèges liés à la modernité qu'offrent les géants superstratiques que sont le français et l'anglais. Ils ne restent pour autant pas repliés sur eux-mêmes, car on perçoit aussi des signes d'ouverture au monde extérieur, comme le montrent des emprunts aux langues africaines comme le nouchi ivoirien.

\subsubsection{L'emprunt au nouchi}

Très captivés par tout ce qui a le vent en poupe, les jeunes vont jusqu'au-delà des frontières du triangle national pour emprunter des termes qui selon eux font ressortir et traduisent leur jeunesse et leur ouverture aux autres cultures. Et ces derniers temps, les Ivoiriens se font beaucoup remarquer à travers leurs styles et leur musique. Voilà pourquoi on peut entendre un jeune garçon dire à ses camarades :

9. A say I go club yesterday, a dance yorobo so téh all man faroté mi for dé. (Hier j'ai tellement bien dansé le yorobo en boîte de nuit que tout le monde m'a récompensé en me donnant de l'argent.)

10. Na laï. Mapouka and zouglou dé out of date now. (C'est faux. Le mapouka et le zouglou sont démodés à présent.)

Yorobo vient du nouchi ivoirien et désigne des pas de danse dont le créateur est l'artiste DJ. Arafat. Ces pas de danse particulièrement athlétiques emportent l'adhésion des jeunes Camerounais en général. Quant à faroté, il vient aussi du nouchi et traduit le fait de donner de l'argent à quelqu'un. Et puisque les domaines de la musique, bien plus de la danse et de l'argent intéressent bien les jeunes, rien de plus normal qu'ils adoptent ces termes. Et s'il fallait traduire cet extrait en pidgin normal, on dirait: A go for night club yesterday, I dance ivorian dance so téh all man dash/give me money. Pour ce qui est de mapouka et zouglou, ils désignent simplement d'autres rythmes musicaux d'origine ivoirienne. En plus du nouchi, les langues nigérianes sont également un réservoir dans lequel puise le Pidgin-English jeune.

\subsubsection{L'emprunt aux langues nigérianes}

La proximité géographique, linguistique, l'invasion des films nigérians, bref, plusieurs facteurs convergent pour amener les anglophones du Cameroun à entrer en contact avec les populations nigérianes et pour que ce contact se fasse sentir à travers des emprunts. L'imitation afin de paraître à la mode étant très développée chez les jeunes, ces derniers opèrent des emprunts aux langues nigérianes. Voilà pourquoi au lieu de dire par exemple 
en pidgin basique à un conducteur de moto taxi lui offrant ses services : Mi a not fit clim machine/moto bike/cycle, A di créze?, une jeune femme préférera le dire de cette autre façon:

11. Mi a no fit clim okada, A di kolo (for ma head)? (Suis-je folle pour emprunter une moto?)

12. A say di adjèbota ngah hi own show skin don do much. (Je dis que la vantardise cette fille de riches va au-delà de toute expression/est exagérée).

On repère alors des expressions étrangères à ce système linguistique tels okada, kolo et adjèbota qui viennent du Nigéria et qui signifient respectivement moto-taxi; folie, devenir fou et choyé, riche, gâté, aisé.

Tel qu'on peut le voir, les jeunes de Buea et de ses environs empruntent à une multitude de langues pour étoffer leur Pidgin-English lorsqu'ils sont entre eux. Ceci dans l'ultime but de faire jeune et de se différencier ainsi du reste de la population pidginophone. Mais toujours est-il que ces emprunts ont des incidences sur la prosodie lorsqu'ils sont prononcés.

\subsection{La prosodie et la phonologie}

Si l'emprunt est un trait caractéristique du Pidgin-English jeune, un autre trait s'observe dans les domaines phonologique et prosodique. En effet, en fonction des sujets abordés et surtout de la provenance des emprunts, les prononciations divergent. Lorsqu'il s'agit par exemple des emprunts au français, on constate que les lexies empruntées ne sont généralement pas accompagnées de leur prononciation native. La lexie est bien française, mais elle est hybridée par une prononciation suivant les rouages du système linguistique pidignophone. Ainsi, pour les termes cinquante mille, forcer, cartouche, démarcheur pris dans les illustrations ci-dessous, on aura les équivalents Pidgin-English jeune /sckãg mil/, / fokse/,/katuch/,/demachsr/:

13. A buy di kouentinini for cinquang mil (J'ai acheté cette kouentinini à cinquante mille)

14. Love na by force? You di fokcé na wéti (L'amour ce n'est pas la force, pourquoi forces-tu?)

15. They cash ma friend wét katouch inside examination class (Mon ami a été attrapé avec des cartouches en pleine salle d'examen)

16. You know that French man? Na yi bi démarchèr for that w'u business (Connais-

tu ce francophone, c'est lui le démarcheur dans notre affaire.)

En général au Cameroun, le Pidgin-English assume une fonction identitaire. Il ne suit pas les normes toujours trop rigides des langues étrangères institutionalisées, et participe à la promotion d'une idiosyncrasie camerounaise, en déterminant une connivence spéciale entre ses locuteurs. C'est ce qui explique que les jeunes ne prennent à ces langues que ce qui les intéresse et qu'ils peuvent adapter à leurs goûts.

En outre, le débit des locuteurs pidginophones jeunes est également très saccadé et rapide, comparé à celui du reste des locuteurs pidgin. Cette rapidité dans la prononciation crée souvent des abréviations dans l'expression, qu'un pidginophone âgé aurait de la peine à comprendre. Ainsi, alors qu'une grand-mère prendrait tout son temps pour demander :

17. You di dou na wéti ? ou encore Ma papa want sé méke mi a die ;

41 Un jeune contractera les mots et dira plus rapidement et en moins de temps:

You-i-dou-na-éti ? Ma pah want sé ma-a-die . 
Une autre particularité de la prosodie du Pidgin-English jeune est qu'elle suit volontiers l'accent nigérian. Le plus évident est l'usage d'un accent mélodieux, et surtout la fréquente utilisation de l'interjection oh! dans une conversation entre proches, surtout lorsque l'on traite des sujets liés aux activités quotidiennes profanes ou non formelles. En voici une illustration de la conversation entre Reine et Laurelle, deux élèves :

18. Laurelle: A say ma friend you lost oh! Why you lost so ? (Mon amie tu deviens si rare! Pour quelle raison?)

Reine: I day oh, massa na poor oh, na poor want kill man oh! (Je suis là, c'est juste que j'ai des soucis financiers)

Laurelle: No be only for you oh, na for all side oh! (Tu n'es pas seule à avoir ces soucis, c'est un problème général.)

Oh, interjection vide de sens ici est très présente et assure une fonction phatique. Elle rapproche davantage les deux jeunes interlocutrices et maintient la proximité entre elles. Les paroles s'accompagnent, comme c'est d'ailleurs perceptible dans les films nigérians, d'une kinésique prononcée (claquements de doigts en rond sur la tête lorsque l'on dit «God forbid »). Cette kinésique propre aux Nigérians est d'autant plus présente dans les manières des jeunes Camerounais anglophones que les chaînes de télévision diffusent à longueur de journée des films nigérians, qui même de façon passive enracinent la gestuelle dans leur subconscient.

En bref, l'accent des jeunes pidginophones est pluriel, en fonction des sujets qu'ils abordent, ils puisent dans diverses sources pour alimenter leur discours. Au-delà de la prosodie, les modifications sémantiques s'opèrent également dans le Pidgin-English des jeunes anglophones.

\subsection{Les changements sémantiques}

Par changement sémantique, on entend le développement d'une signification nouvelle dans le cadre d'un segment phonologique. En plus des néologies formelles telles que les emprunts et la prosodie mentionnées plus haut, les jeunes des zones anglophones en général opèrent aussi des changements de sens pour exprimer leurs manières de vivre et de penser. Pour cela, ils donnent aux termes déjà existants des sens nouveaux ou différents de leurs origines et qui cadrent mieux selon eux avec leurs réalités. Les paroles de ce locuteur qui informe son camarade de sa rupture avec sa petite amie en donne une idée :

19. Ma man no bi ma ngah don nock mi yesterday? Mi-an-yi don bolè ! (Ma petite ami m'a quittée hier. C'est terminé entre elle et moi).

6 L'item nock qui habituellement veut dire cogner signifie dans ce contexte éconduire, rompre avec. Et l'expression nock mi relève du Pidgin-English jeune d'autant plus qu'en pidgin courant, on aurait dit Mi and ma girl friend we don finish / seperate.

On a affaire à un changement de connotation, dans la mesure où le sens propre de départ est différent du nouveau. Un autre glissement de sens peut aussi s'observer dans la suite mettant en scène Nnane et Njoke :

20. Nanne: You di go ékol? (Tu vas à l'école?)

Njoke : Confirm! (oui !)

Confirm a changé sa signification de corroborer, renforcer, pour signifier à présent oui ou yes, qui est plutôt une affirmation et une réponse à l'interrogation directe précédemment formulée par Nnane. 


\section{plus nette :}

21. Gizo : A bi no! Na all ya own that you no fit think about different thing, but maa-tell you éh, A need na only one milky baby then ma own correct! (Je le savais! Tu ne peux penser à autre chose ; mais que je te dise ; j'ai juste besoin d'une jolie fille et mon compte est bon)

Pikolo : You siam? A bi tell you séh you go die woman one day. Déh di tchop baby them? If mi a go déh nor, a si ma mounching, a di mounch without stress. (Tu vois, je t'avais dis que ta mort viendrait des femmes. Les mange-t-on? Moi si j'y vais, je trouve de la nourriture, je vais manger sans pression).

Comme on peut le relever, le terme anglais baby emprunté par le Pidgin-English jeune dans l'extrait n'a plus rien d'un bébé ou d'un enfant qui renvoie à son sans anglais originel. Il signifie désormais filles ou femmes et Pikolo l'a bien confirmé après dans son reproche à son ami en disant:You go die woman them one day. Le changement de connotation est une tactique assez productive dans le Pidgin-English jeune. Parfois aussi, il est juste question d'être à la mode et de suivre la mouvance jeune. Les expressions idiomatiques poursuivent également les mêmes objectifs.

\subsection{Les expressions idiomatiques}

51 Une expression idiomatique est une expression propre à une langue et qui n'a pas nécessairement d'équivalent littéral dans d'autres langues. En d'autres termes, cette notion renvoie à l'ensemble des locutions perçues comme figées par les usages d'une langue, et dont la signification tient à une mémorisation préalable. Il pourrait aussi s'agir de locutions propres à une langue et qu'il serait difficile de traduire. En peu de mots, nous parlons ici des camerounismes qui sont des expressions propres aux usages camerounais. C'est dans cet ordre d'idées que va la démonstration ci-après :

22. A say with MTN you talk for njoh sooooo téhhhh. A say na njoh this!? (Avec le réseau de téléphonie mobile MTN, tu parles à n'en plus finir. Quelle gratuité!!!)

Un locuteur âgé du pidgin se serait arrêté à la première phrase. Mais pour suivre la tendance, un jeune ajoute l'interrogation rhétorique na njoh this? qui n'est en fait que la confirmation ou l'emphase sur ce qu'avec MTN on communique presque gratuitement.

On pourrait également repérer dans les illustrations suivantes, une formule toute faite :

23. Petit-Pays sing for foire yesterday, they faroté hi so téeeh. A say na money you bi want siam? (Petit Pays Pays a chanté hier à la foire. Ses admirateurs lui ont donné tellement d'argent).

24. We go beach last week, a say na sexy ngah you want siam ? (On est allé à la plage la semaine dernière : Il y avait des filles super sexy.

Les occurrences portent des interrogations toujours rhétoriques, puisqu'au lieu de demander des informations, elles affirment et confirment plutôt des faits : c'est-à-dire que Petit-Pays a reçu beaucoup d'argent à la foire, ou encore qu'ils y avait des filles vraiment sexy à la plage. Les deux derniers exemples sont également des calques, car ils sont les traductions littérales du camerounisme tiré des langues locales très en vogue actuellement en français C'est....... que tu/vous veux/voulez voir?

On peut donc déduire que ces usages relèvent des effets de style dans le langage régis par des normes et cultures endogènes que les jeunes désirent mettre en exergue. Il s'agit aussi d'un désir de se sentir jeune, de se hisser à la mode et de suivre le courant de la 
nouveauté. En dehors des expressions idiomatiques, les calques méritent aussi qu'on leur accorde une attention.

\subsection{Les calques}

Selon Dubois et al. (2001: 127), les calques linguistiques sont des utilisations d'unités lexicales d'une langue dans l'arrangement structural d'une autre langue (Dubois et al. 2001 : 127). De là, on peut dire que le calque se rapproche de l'emprunt, dans la mesure des éléments d'un système linguistique étranger se retrouvent dans une autre langue. Néanmoins, il demeure un emprunt partiel et se différencie de la sorte de l'emprunt original, dans la mesure où contrairement à cet emprunt qui transfère à la fois le signifiant et le signifié d'un terme, le calque se limite à l'emprunt incomplet d'un signifié étranger au détriment de son signifiant. Ce processus de formation est aussi utilisé par les jeunes pidginophones. C'est le cas des exemples de la rubrique précédente.

57 En effet, en plus d'être des expressions idiomatiques, les tournures interrogatives na sexy ngah you want siam? et na money you want siam ? sont également des calques. En fait, la variété utilisée ici pour traduire cette réalité est le Pidgin-English, mais le sens originel de ces énoncés n'est pas pidgin. Il a été emprunté de l'expression en français C'est....... que tu / vous veux / voulez voir? elle-même empruntée aux langues maternelles du pays qui possèdent toutes ou presque, une formule similaire.

Un autre exemple de calque est ce titre que l'on peut lire sur une affiche destinée aux jeunes étudiants de l'université de Buea en particulier, qui du reste sont friands des fêtes :

25. Freshmen djoka!!!!!!! (fête des hommes chics).

Le calque ici repose sur le terme fresh qui est calqué sur le mot connoté français frais qui signifie alors élégant, bien habillé, charmant, mais dont il a été rejeté la graphie pour ne retenir que la connotation. Car fresh ici n'est pas le contraire de chaud et n'est pas dans son sens courant de nouveau, neuf ou récent. Il réfère bel et bien à l'élégance, au chic. On pourrait rendre cet exemple par djoka = soirée / fête (tiré du bassa) des jeunes/hommes chics, élégants, distingués. Ainsi, l'expression appartient bien à la variété pidgin jeune, dans la mesure où le pidgin courant se serait contenté de dire fine man/people party.

60 Ainsi, les calques en général et ceux des langues locales transposés dans le pidgin english en particulier confirment le rôle identitaire que joue les pidgin english jeune, et qui par ailleurs recourt à d'autres procédés linguistiques au rang desquels apparaît aussi la périphrase.

\subsection{La périphrase}

61 La périphrase se classe aussi parmi les signes qui distinguent le pidgin jeune du pidgin courant. La périphrase exprime par plusieurs mots une notion qu'un seul ou alors peu de mots pourraient exprimer. Autrement dit, il s'agit des détours dans le langage ou encore des circonlocutions. Les jeunes sont adeptes de ces tournures que l'on peut repérer dans l'exemple suivant:

26. A say ma old man bit me today so tééh ! (Mon père m'a bien battu aujourd'hui !)

62 L'expression détournée old man emprunté à l'anglais peut être simplement traduite en pidgin courant par papa ou pah qui signifie père. 
63 De même, cet autre exemple présente aussi la circonlocution na ya man this de Gizo, alors qu'un simple fine en pidgin simple aurait suffi pour passer le message à son ami Pikolo, car il suppose que s'il n'allait pas bien, son ami n'allait pas le voir dans l'état où il se trouve. Tel qu'on peut le constater, les jeunes se servent d'expressions indirectes afin d'être beaucoup plus éloquents.

\subsection{Les onomatopées}

L'analyse du corpus permet d'observer que parfois, au lieu de nommer les réalités auxquelles ils voulaient faire référence, les jeunes préfèrent plutôt imiter les sons de la chose dénommée. Ce faisant, ils créent des néologies par onomatopée. De toute évidence, il est question pour eux d'être le plus convainquant. Quelques extraits illustrent ce fait.

Une jeune étudiante en colère raconte à son amie comment un garçon qu'elle déteste a eu le toupet de lui faire la cour.

27. A say a wan siam that crézz boy waka kougna-kougna cam tell mi sé hi love mi, I wan kolo for vex! (Figures-toi que ce crétin a marché kougna-kougna pour venir me dire qu'il m'aime. J'allais mourir de colère.)

Un autre dit à son compère qu'il souffre du choléra en ces termes :

28. Massa a di sick oh ! bpouh-pvroup want kil-me. (Gars, je suis malade. Le choléra veut me tuer)

\section{Un autre encore admire le téléphone de son ami en disant}

29./ This ya ting-ling ting-ling na war oh, m'a-a siam! (Ton téléphone est très beau, puis-je le voir de près?)

Le terme kougna-kougna, est un mot imitatif. Il aurait pu être omis en pidgin courant et imite la démarche de la personne dont parle la jeune fille. Pour ce qui est de bpouh-pvroup, en pidgin courant, c'est une des néologies les plus récentes au Cameroun. Elle est née avec les récentes vagues d'épidémies de choléra dans diverses régions du pays, dont les symptômes principaux se résument aux vomissements et à la diarrhée. Ce terme est formé grâce au processus de composition, le premier terme, bpouh reproduit le son que produit quelqu'un qui vomit, tandis que le second, pvroup rend le son que produit une personne atteinte de diarrhée. De la sorte, bpouh-pvroup désigne le choléra qui se signale par la diarrhée et les vomissures.

Enfin, ting-ling ting-ling, qui du reste présente des signes d'un emprunt approximatif du terme anglais tinkle, imite la sonnerie d'un téléphone et par extension, le téléphone luimême.

L'imagerie dans les calques, périphrases et onomatopées, est très productive chez les jeunes pidginophones, et même les plus âgés qui font rapidement le lien avec ce dont ils parlent.

\subsection{La dérivation suffixale}

71 Par dérivation, Dubois et al. (2001: 1236) désignent « le processus de formation des unités lexicales. » Ce processus de formation se caractérise par l'adjonction d'affixes au radical d'un mot. On obtient ainsi de nouveaux mots par préfixation, parasynthétisation ou suffixation, selon que les affixes sont placés avant, après ou derrière le radical. L'usage de la préfixation et de la parasynthétisation ne semblent pas trop intéresser les jeunes 
pidginophones. Par contre, ils nourrissent un faible pour la suffixation. On peut le constater dans cet extrait où une étudiante parle des ses performances scolaires à son ami :

30. - A hear séh results dé out. You don validé dah French this time? (J'ai entendu que les résultats ont été publiés. As-tu validé l'UV de français cette fois?)

- For who site? A don echouéam again, ma sister a go kam validéam one day? (Non, j'ai encore échoué. Ma sœur, vais-je la valider un jour?)

32. - If mi a go déh nor, a si ma mounching, a di mounch without stress. (Moi si j'y

vais et je trouve de la nourriture, je vais manger sans pression).

Tel qu'il est perceptible avec les items échoueam, validéam et mounching (altération de manger), les radicaux français -échoue, -validé et -manger ont été hybridés à l'aide des suffixes verbaux passe partout pidgin -am et anglais -ing placés à la droite de ces radicaux pour former de nouveaux termes et enrichir ainsi leur parler. Ce type de formation est spécifique aux jeunes pidginophones, d'autant plus qu'en pidgin courant on aurait pu les remplacer respectivement les termes A no passam/ a fail/ tchop. Ils se servent de radicaux français, sans doute pour montrer qu'ils sont instruits et ont une connaissance, ne seraitce que sommaire du français qui est leur deuxième langue officielle, même si pour assumer leur identité de camerounais, ils le mélangent au pidgin, à l'anglais et aux langues locales et ne respectent plus de ce fait plus les règles de grammaire. Et qui plus est, les sujets qu'ils traitent sont en relation avec la jeunesse, puisqu'ils parlent le plus de l'école, des sorties, de la nourriture, etc.

\subsection{L'aphérèse}

Tout comme l'apocope, l'aphérèse est une variante du système abréviatif de mots par troncation. La troncation en elle-même "consiste à supprimer les syllabes d'un mots», d'après Dumont et Maurer (1995: 36). A l'opposé de l'apocope qui s'opère à la fin d'un mot, l'aphérèse est la chute d'un ou de plusieurs phonèmes en début d'un mot. Il a été observé que certains termes du Pidgin-English apparaissaient sans leurs syllabes initiales. A titre indicatif, on a les occurrences qui suivent.

A ses amis une étudiante dit :

32. The way that french madam di nervé mi hein, na papa God know (Cette dame de français m'énerve à en mourir.)

Un autre veut connaître la raison de l'absence de son ami, et il répond

33. A- Why you no kam écol last week ? (Pourquoi n'étais-tu pas à l'école la semaine dernière?

B- A bi go visit ma onkou for ngola. Na yi dash mi di ma small modern phone. (Je suis allé rendre visite à mon oncle à Yaoundé, C'est lui qui m'a offert ce petit téléphone sophistiqué).

34. A get for go club today, na why a déh happy/di glad (Je vais aller en boîte de nuit aujourd'hui, c'est pourquoi je suis content)

76 Nervé apparaît dans le premier exemple sans le é- du début. Pourtant, c'est bien le mot énervé que l'étudiante veut prononcer. On peut mettre cela au compte d'un défaut de prononciation et de la méconnaissance de l'orthographe et la prosodie exactes de ce mot. L'essentiel pour elle est de faire voir à ses amis pidginophones qu'elle connait ce mot français.

77 Ngola en réalité Ongola (Yaoundé) vient de l'éwondo et transite par le camfranglais avant de se retrouver dans le pidgin english jeune. Ici, il apparaît dépourvu de son $O$ initial. Il en 
est de même pour phone qui lui, est plus connu sous cette forme du reste déjà attestée en anglais, et que la majorité préfère au mot entier plus long, à savoir téléphone. L'usage de ce dernier mot par les jeunes anglophones varie en fonction du sujet traité, des relations qu'entretiennent les locuteurs en présence. Car pour des raisons d'expressivité (surtout identitaires) les jeunes préféreront l'onomatopée ting-ling ting-ling à phone qui sonne plus pidgin et qui est plus vulgaire. On voit aussi que club apparaît sans sa particule gauche de composition night qui donne à ce mot son sens plein. Cet usage est d'ailleurs naturalisé aussi bien en français qu'en anglais, au point qu'il mène une vive concurrence au terme d'origine night club.

\subsection{Les termes transcatégorisés}

78 La transcatégorisation est un phénomène de conversion, c'est-à-dire par lequel un mot est capable de quitter sa catégorie grammaticale initiale pour occuper une autre. Le phénomène de changement de classe grammaticale se repère dans les exemples suivants.

$\mathrm{Au}$ cours d'une scène de salutation, un interlocuteur informe son ami de sa maladie comme ceci :

35. A- Na how this man?

B- Massa, A dé maladie,

80 Un autre répond par la positive à son ami à la question de savoir s'il a payé sa scolarité ainsi :

36. Confirm!

81 Maladie est originellement un nom. Ici, il est employé où il pu être remplacé par un adjectif. Pour ce qui est de confirm, il renvoie au verbe to confirm, qui veut dire " confirmer ». Mais dans le présent contexte, il quitte sa classe grammaticale initiale de verbe pour rejoindre celle d'adverbe. En effet, confirm renvoie en réalité à l'adverbe d'affirmation yes, c'est-à-dire « oui », ou encore de la locution adverbiale of course « bien sûr ».

82 Ce genre d'usage est aussi une exclusivité du Pidgin-English jeune, car en pidgin normal, on aurait l'une des expressions A no well/A di sick/ A no dé fine pour transmettre le même message, à savoir qu'il est malade. De même, le terme Yes emprunté à l'anglais et qui transite par le pidgin courant avant d'atterrir dans le pidgin jeune aurait remplacé confirm. Mais on peut comprendre ce phénomène lorsque l'on sait que pidgin est une langue identitaire de la place publique camerounaise qui se singularise avant tout par son extrême souplesse. C'est pourquoi, les jeunes se plaisent à la parler au gré de leurs fantaisies. En plus, le pidgin qui est une langue de grande communication essentiellement orale, favorise cet état de choses. Le fait qu'un jeune locuteur du Pidgin-English connaisse par exemple le vocables français maladie suffit pour lui, pour le réutiliser à temps et à contre-temps. Qu'importe, si de toutes les façons, son message est décodé correctement par ceux avec qui il interagit. Et, de plus en plus, de telles formations et bien d'autres naissent de façon consciente ou pas de l'imagination des jeunes épris d'un désir de marquer leur temps et leur présence dans un pays où la guerre des langues est plus que jamais effective.

83 Avant de clore ces analyses attardons-nous sur les représentations que les jeunes anglophones surtout nourrissent à l'égard du leur Pidgin-English. 


\section{Les représentations dans le Pidgin-English jeune} d'images, des positions idéologiques, des croyances qu'ont des locuteurs à propos des langues en présence et des pratiques linguistiques, les leurs et celles des autres ». Dans cet ordre d'idées, cette section a pour objectif de comprendre au mieux les raisons et les motivations des jeunes pidginophones dans le développement de cette variété de langue. Il est question ici de comprendre vers quels idéaux langagiers ces jeunes veulent tendre. A ce titre, nous pouvons essayer de savoir pourquoi dans une zone anglophone comme Buea en particulier, où le Pidgin-English s'impose comme étant le véhiculaire identitaire créant connivence et rapprochement, les jeunes créeraient encore un parler spécifique. Le pidgin étant formellement interdit dans les lieux institutionnels, comme les campus scolaires, la quasi-totalité du public cible a souhaité s'exprimer en anglais dès lorsqu'il a été question d'enregistrer leurs avis. Les réactions suivantes ont été recueillies :

a) "With Youth Pidgin-English you look interesting".

b) "It makes us feel really young"

c) "With this pidgin, we are proud and feel free"

d) "We use that new pidgin in order to secure some of our communication"

e) "We are still young, so we are following the tendency by speaking that way"

f) "Youth Pidgin-English permit us here to distinguish ourselve from the rest of the population."

g) "It is a way of feeling young and be proud of it".

h) "I use Youth Pidgin-English when I desire to be more eloquent and emphasize on something in a conversation with my friends"

i) "Youth Pidgin-English is spoken to put ambiance in our communication."

j) Among youth, youth Pidgin-English shows our own style and fashion and all innovations youth are capable of."

k) "We use Youth Pidgin-English to make fun and keep people happy while transmitting a message".

Il ressort de ces interventions l'idée centrale selon laquelle le Pidgin-English jeune existe, qu'il est effectivement parlé et qu'il est un parler créé par les jeunes pour les jeunes, dans l'optique de répondre exclusivement à leurs besoins. C'est la raison principale pour laquelle les jeunes dans l'ensemble trouvent que ce parler leur procure une fierté, les rend libres et les distingue des autres couches de la population.

En outre, cette variété a également du crédit au sein de la population cible parce que son extrême malléabilité autorise de nombreuses images, circonlocutions et autres tournures qui participent de véritables stratégies oratoires. Il donne la possibilité au jeune de s'exercer intellectuellement, de mettre son imagination à contribution pour créer et faire tout le temps des innovations qui enchantent ses interlocuteurs, car elles permettent de facilement emporter l'adhésion de l'auditoire, tout en l'amusant et en le maintenant de ce fait en haleine; d'où l'idée d' "ambiance » qu'a mentionné un locuteur-enquêté. Cette ambiance permet donc de se détendre ( «make fun» comme disaient certains). Et ce faisant, il demeure continuellement dynamique et à la mode, car l'anxiété qui régit les langues normées n'existe pas ici. Le jeune se sent libre en l'utilisant, parfaitement à son aise et sans stress.

Enfin, certains jeunes trouvent en ce type de pidgin un moyen de sécuriser leur communication devant les personnes non initiées ou non immergées dans ce parler. Et là, l'on comprend vite que c'est peut-être pour mettre les plus âgées à l'écart de leurs 
conversations privées en rapport par exemple avec sujets portant sur le sexe, les sorties clandestines, les mauvaises compagnies, les mauvaises notes, etc.

\section{Conclusion} anglophone est la conséquence du phénomène de contact entre les nombreuses langues au Cameroun. L'évolution pourrait également y être pour quelque chose, car de peur de paraître vieux jeu, les jeunes veulent sans cesse se hisser à la pointe du modernisme. Ainsi, ayant trouvé le Pidgin-English courant désuet et caduque pour traduire les réalités du monde nouveau, ils ont opté pour ce nouveau parler qui reflète mieux leur identité camerounaise, leur vécu et leurs aspirations.

Pour mieux connaître cette variété, un bref exposé sur le Pidgin-English dans l'ensemble du pays a été fait, avant d'examiner celui de la zone anglophone du Cameroun. Ensuite, ont été passés au peigne fin les traits distinctifs de ce parler qu'affectionnent les jeunes en zone anglophone. C'est alors qu'on a repéré comme en faisant partie, les termes empruntés au français, principale langue dominante du territoire, au camfranglais, parler composite des régions francophones, aux langues locales et même aux langues d'autres pays d'Afrique, à l'instar du Nigéria et de la Côte d'Ivoire. Ces emprunts sont accompagnés de prosodies variées et particulières, caractéristiques des contacts humains, culturels et linguistiques entrepris. En outre, des indices relevant des mutations sémantiques des termes, des expressions idiomatiques, des calques, des périphrases, des onomatopées, de la dérivation, de l'aphérèse et de la transcatégorisation, ont témoigné de la volonté des jeunes anglophones d'employer une pluralité de processus de créations de nouveaux termes, afin d'alimenter, enrichir et diversifier leur réservoir lexicologique, sémantique, syntaxique, phonétique et phonologique, etc, et de le maintenir continuellement à jour. Une fenêtre a enfin été ouverte sur les représentations que se font les jeunes de ce parler, pour davantage comprendre leurs motivations et idéaux par rapport à ce Pidgin-English. Quant à savoir s'il fera long feu ou pas, seul l'avenir nous le dira.

\section{BIBLIOGRAPHIE}

AYAFOR, M. (2005) «Is pidgin facing death or gaining ground in Cameroon », A paper presented at the international conference on language, literature and identity, Yaounde.

CALVET , J-L. (1999) Pour une écologie des langues du monde, Paris, Harmattan.

CHUMBOW, B.S. et A.S. BOBDA (2000) «French in West Africa : a sociolinguistic perspective », International Journal of the Sociology of Language, 141, 39-60.

DUBOIS, J. et alii, (2001) Dictionnaire de linguistique, Paris, Larousse-Bordas/AER. 
DUMONT , P. et MAURER, B. (1995) Sociolinguistique du français en Afrique francophone, Vanves, Hachette.

ECHU, G. The language question in Cameroon, Trans, http://www.linguistik-online.de/18_04/ echu.html (consulté oct. 2015)

FAME NDONGO, J. (1996) Un regard sur la communication. À la découverte de la géométrie, Yaounde, Saint Paul.

FERAL, C. de (2009) « Nommer et catégoriser des pratiques urbaines : pidgin et francanglais au Cameroun ", in Féral, C. de (dir.), Le nom des langues en Afrique sub-saharienne : pratiques, dénominations, catégorisations. Naming Languages in Sub-Saharan Africa : Practices, Names, Categorisations, Louvain-la-Neuve, Peeters, BCILL 124, 119-152.

FÉRAL, C. de (2004) « Français et langues en contact chez les jeunes en milieu urbain : vers de nouvelles identités ", Penser la francophonie; concepts, actions et outils linguistiques, Actes des premières Journées scientifiques communes des réseaux de chercheurs concernant la langue, Ouagadougou (Burkina Faso), 31 mai-1er juin 2004, Paris Editions des Archives Contemporaines, 583-597.

FÉRAL, C. de (1989) Pidgin-English du Cameroun : description linguistique et sociolinguistique, Paris, Peeters/Selaf.

GUILBERT L. (1975) La Créativité lexicale, Paris, Larousse.

KOUEGA, J-P. (2001) « Pidgin facing death in Cameroon », Terralingua, discussion paper $n^{\circ} 7$.

LABOV, W. (1976) Sociolinguistique, Paris, édition de Minuit.

MBANGWANA, P. (1983) «The scope and role of Pidgin English in Cameroon », EDNA, L.

NGEFAC A. \& SALA, B. (2006) « Cameroon Pidgin and Cameroon English at a confluence », English World-wide, 217-227.

PIEBOP, C. M. G. (2014) Contact de langues et appropriation du français dans l'œuvre romanesques de Camille Nkoa Atenga, Thèse de doctorat $\mathrm{PhD}$, Université de Yaoundé $\mathrm{I}$.

SALA, B. M. (2009) «Writing in Cameroon Pidgin-english : begging the question, in English Today, Volume 25, 02, 11-17, publié en ligne le 26 Mai 2009 : http://journals.cambridge.org/action/ displayIssue?jid=ENG\&volumeId=25\&seriesId=0\&issueId $=02$.

SALA, B. (2005) Aspects of the Cameroon English sentence, PhD dissertation, University of Yaounde I. SCHRÖDER, A. (2003) Status, functions and prospects of Pidgin English. An empirical approach to language dynamics in Cameroon, Tubingen, Narr.

SIMO BOBDA, A. \& H. G. WOLF. (2003) « Pidgin-English in Cameroon in the new millennium", in LUCKO P., LOTHAR P. \& H-G. WOLF (eds) Studies in African Varieties of English, Frankfurt a. M. Peter Lang, 101-117.

\section{NOTES}

1. G. Echu, The language question in Cameroon, in Trans, http://www.linguistik-online.de/18_04/ echu.html. 


\section{RÉSUMÉS}

La cohabitation du Pidgin-English et de plusieurs autres langues dans et hors du Cameroun a abouti à l'émergence d'une variété de pidgin dite "jeune" dans les régions anglophones et particulièrement à Buea. La présente étude se donne pour objectif de passer au crible les singularités de ce nouveau parler. Il apparaît que, sur la base du Pidgin-English ou kamtok, il se démarque à travers de nombreux emprunts faits au français, au camfranglais, aux langues maternelles locales et même aux langues nigérianes et ivoiriennes. En outre, plusieurs termes subissent des transformations au niveau de la prosodie, de la morphosyntaxe (dérivation, transcatégorisation...) et même de la sémantique (expressions idiomatiques, calques, périphrases, glissements sémantiques, onomatopées.) La charpente de cette étude est fournie par l'approche sociolinguistique. Les données, quant à elles, ont été recueillies à travers des interviews et des productions orales et écrites des jeunes, principalement ceux des institutions scolaires de Buea.

Due to the contact between Pidgin English and many languages in and out of Cameroon, a socalled "youth" Pidgin-English has emerged in English-speaking regions and especially in Buea. This paper examines the various characteristics of that new language. A close scrutiny of that language shows that based on the current kamtok, it contains a lot of borrowed lexical items from Cameroonian and even foreign languages. Many words and expressions undergo several modifications at the level of phonology / intonation / pronunciation, morphosyntax (derivation, change of grammatical category) and semantics (loan translation, idiomatic expressions (word express), transfer, periphrasis, onomatopoeias, etc.) The sociolinguistic approach in general as been adopted to carry out analysis and the corpus gathered for this study has been collected through interview and participant observation from young pidgin speakers, mostly students in Buea.

\section{INDEX}

Mots-clés : plurilinguisme, Pidgin-English jeune, identité, région anglophone, particularités.

Keywords : plurilingualism, youth pidgin English, identity, Anglophone region, particularities

\section{AUTEUR}

\section{CÉPHANIE MIRABELLE GISÈLE PIEBOP}

Bilingual Grammar School Molyko-Buea et Université de Buea

giselepiebop@live.fr 